\title{
Ressonância magnética da osteonecrose do joelho: estudo de 19 casos*
}

\author{
Magnetic resonance imaging of knee osteonecrosis: a study of 19 cases \\ Daniel Leme da Cunha ${ }^{1}$, Antonio Carlos Pires Carvalho ${ }^{2}$, Elísio José Salgado Ribeiro ${ }^{3}$, \\ Romeu Côrtes Domingues ${ }^{4}$
}

Resumo OBJETIVO: Descrever os achados epidemiológicos, clínicos e de ressonância magnética da osteonecrose das porções distal do fêmur e proximal da tíbia. MATERIAIS E MÉTODOS: Avaliação de 19 pacientes (12 mulheres e 7 homens), sem história prévia de fatores causais, com achados à ressonância magnética sugestivos de osteonecrose do platô tibial ou côndilo femoral. RESULTADOS: Verificou-se a presença de anormalidades osteocondrais em $63,1 \%$ dos casos e em $73,6 \%$ destes houve associação com lesão meniscal ipsilateral. Houve também importante associação com edema na medular óssea em correspondência (grau III em 16 casos). CONCLUSÃO: A ressonância magnética demonstrou ser um método não invasivo com boa sensibilidade no diagnóstico da osteonecrose do joelho, bem como das lesões associadas, sendo mais frequente nas mulheres $(63 \%$ dos casos).

Unitermos: Osteonecrose; Imagem por ressonância magnética; Anormalidades subcondrais.

Abstract OBJECTIVE: To describe epidemiological, clinical and magnetic resonance imaging findings of osteonecrosis in the distal femur and proximal tibia. MATERIALS AND METHODS: Evaluation of 19 patients (12 women and 7 men), with no previous history of causative factors, with magnetic resonance imaging findings suggestive of osteonecrosis in the tibial plateau or femoral condyle. RESULTS: Osteochondral abnormalities were observed in $63.1 \%$ of the cases; in $73.6 \%$ of them, such abnormality was associated with ipsilateral meniscal lesion. Also, a significant association with bone marrow edema (grade III in 16 cases) was observed. CONCLUSION: Magnetic resonance imaging has demonstrated to be a noninvasive method with good sensitivity in the diagnosis of knee osteonecrosis as well as of associated lesions which are most frequently found in women (63\% of cases).

Keywords: Osteonecrosis; Magnetic resonance imaging; Subchondral abnormalities.

Cunha DL, Carvalho ACP, Ribeiro EJS, Domingues RC. Ressonância magnética da osteonecrose do joelho: estudo de 19 casos. Radiol Bras. 2010;43(2):77-80.

\section{INTRODUÇÃO}

A osteonecrose espontânea do joelho é tipicamente encontrada em pacientes do sexo feminino, da raça branca, na sexta e sétima décadas de vida, caracterizada por

\footnotetext{
* Trabalho realizado na Faculdade de Medicina da Universidade Federal do Rio de Janeiro (UFRJ) e nas Clínicas de Diagnóstico Por Imagem (CDPI) e Multi-Imagem, Rio de Janeiro, RJ Brasil.

1. Mestrando do Programa de Pós-Graduação da Faculdade de Medicina da Universidade Federal do Rio de Janeiro (UFRJ), Médico Radiologista das Clínicas de Diagnóstico Por Imagem (CDPI) e Multi-Imagem, Rio de Janeiro, RJ, Brasil.

2. Doutor, Professor Adjunto, Coordenador Adjunto do Programa de Pós-Graduação da Faculdade de Medicina da Universidade Federal do Rio de Janeiro (UFRJ), Rio de Janeiro, RJ, Brasil.

3. Médico Radiologista das Clínicas de Diagnóstico Por Imagem (CDPI) e Multi-Imagem, Rio de Janeiro, RJ, Brasil.

4. Médico Radiologista, Diretor das Clínicas de Diagnóstico Por Imagem (CDPI) e Multi-Imagem, Rio de Janeiro, RJ, Brasil.

Endereço para correspondência: Dr. Daniel Leme da Cunha. Rua Miguel de Frias, 77, Bloco 2, ap. 1403, Icaraí. Niterói, RJ, Brasil, 24220-008. E-mail: danielc@predialnet.com.br

Recebido para publicação em 27/9/2009. Aceito, após revisão, em 3/12/2009.
}

gonalgia importante de início súbito, não associada a trauma local, cirurgia meniscal ou mesmo terapia prévia com corticosteroides. Yamamoto e Bullough ${ }^{(1)}$ apontam que, além da suposta origem vascular associada com o aumento da pressão intraóssea, o mecanismo de fratura de insuficiência assumiria papel de fator etiopatogênico relevante, além da osteoartrose, questionando assim o termo "espontânea", uma vez que fatores causais poderiam ser implicados.

O diagnóstico se estabelece a partir da imagem que confirma a suspeita clínica. A radiografia convencional, nas primeiras fases, é normal ou apresenta-se com sutis alterações $^{(2)}$; a ressonância magnética é especialmente útil em confirmar a suspeita do diagnóstico quando os achados da radiologia convencional são normais ou duvidosos, uma vez que demonstra anormalidades da intensidade de sinal sugestivas, quando não características, além de ser um método não invasivo de obtenção de imagens multiplanares com alta definição $0^{(3)}$.

Ramnath e Kattapuram ${ }^{(4)}$ relacionam a presença de uma anormalidade de sinal subcondral "linear" nos casos com suposta origem na fratura por insuficiência, estando esta ausente nos casos de osteoartrose (a qual também apresenta anormalidades semelhantes do osso subcondral relacionadas à osteonecrose).

O objetivo deste trabalho foi descrever os achados epidemiológicos, clínicos e, principalmente, de imagem por ressonância magnética da osteonecrose do joelho.

\section{MATERIAIS E MÉTODOS}

Foram avaliados 19 exames de ressonância magnética do joelho de 12 mulheres e 7 homens que apresentavam sintomas agudos, sem história prévia de fatores causais, e sinais compatíveis com osteonecrose 
no exame, no período de março a dezembro de 2007. Foram excluídos os pacientes com história de trauma, cirurgia local ou uso de corticosteroides prévios. Os pacientes analisados, todos da raça branca, com idades variando entre 45 e 77 anos (média de 61 anos), apresentavam gonalgia, sendo feita análise clínica que levou em conta as seguintes características: o padrão da dor (súbita ou insidiosa, localizada ou difusa) e a associação de edema, bloqueio e instabilidade articulares.

Os exames de ressonância magnética foram realizados em aparelho GE 1.5 T Signa (General Electric Medical Systems; Milwaukee, WI, EUA), sendo obtidas imagens nas sequências pesada em T1 no plano sagital (tempo de repetição [TR] $=405$; tempo de excitação [TE] = 13,9; número de excitações $[\mathrm{NEX}]=1$; echo train length [ETL] = 2) e em densidade protônica com supressão de gordura nos planos coronal $(\mathrm{TR}=1775 ; \mathrm{TE}=14 ; \mathrm{NEX}=2 ; \mathrm{ETL}=4)$, axial $(\mathrm{TR}=1975 ; \mathrm{TE}=21 ; \mathrm{NEX}=2 ; \mathrm{ETL}$ $=4)$ e sagital $(\mathrm{TR}=2250 ; \mathrm{TE}=14 ; \mathrm{NEX}=$ 2; ETL = 4).

As anormalidades do osso subcondral foram avaliadas por dois radiologistas com experiência em sistema musculoesquelético, sendo tais anormalidades definidas como alterações focais da intensidade de sinal, com sinal hipointenso nas imagens pesadas em T1 e sinal variável (hiper ou hipointenso) nas imagens pesadas em T2. A localização de tais achados foi definida da seguinte forma: situadas na extremidade distal do fêmur (côndilo medial ou lateral) ou na tíbia (platô interno ou externo). Posteriormente, dividiu-se arbitrariamente em três zonas cada uma das topografias acima descritas, tanto no plano coronal quanto no sagital, para definir o epicentro da lesão. No plano coronal (Figura 1), a zona 1 seria o terço interno do côndilo femoral/platô tibial, a zona 2 seria o terço médio e a zona 3 , o terço externo. No plano sagital, 1, 2 e 3 seriam, respectivamente, os terços posterior, médio e anterior das regiões estudadas. Verificou-se também a presença ou não de anormalidade "linear" subcondral da intensidade de sinal. Foi realizada ainda a pesquisa de edema na medular óssea, anormalidades na cartilagem articular e lesão meniscal (instável ou não) ipsilaterais associadas. O edema na medular óssea foi graduado de I a III (leve, moderado ou importante, respectivamente). As lesões subcondrais foram mensuradas (em milímetros) nos três planos (longitudinal $\times$ ântero-posterior $\times$ transversal).

\section{RESULTADOS}

Em relação ao quadro clínico, em 14 pacientes $(73,6 \%)$ a dor iniciou-se de forma súbita, enquanto em 5 casos a apresentação da gonalgia foi insidiosa. Oito pacientes relataram bloqueio articular, dois citaram aumento do volume articular (edema) e três queixaram-se de instabilidade.

Todos os pacientes apresentaram lesões com sinal hipointenso em T1 e predominantemente hiperintenso $(89,4 \%)$ nas imagens em densidade protônica com supressão de gordura.

Em oito casos as lesões ocorreram no platô tibial externo, em nove no côndilo femoral medial e em apenas dois pacientes no platô tibial interno. Não houve acometimento do côndilo femoral lateral em nenhum caso. No plano sagital, observou-se maior ocorrência das lesões com epicentro na zona 2 (17 casos; 89,4\%), dois casos na zona 3 e nenhum caso situado na zona 1 . No plano coronal, a maioria dos achados acometeu a zona 3 (11 casos; 57,8\%), seguida pelas zonas 2 (7 casos) e em apenas um único caso a zona 1.

Verificou-se presença de anormalidade linear subcondral (Figura 2) em 15 dos 19 casos $(78,9 \%)$, estando esta ausente nos restantes.

Foi observada associação com edema na medular óssea, sendo grau III em 16 casos e grau II em 3 casos (Figura 3).

O tamanho das lesões variou de 1,5 a 8 $\mathrm{mm}$ no eixo longitudinal, de 2 a $30 \mathrm{~mm}$ no eixo ântero-posterior, e de 6 a $26 \mathrm{~mm}$ no eixo transversal, com médias de 4,5 mm

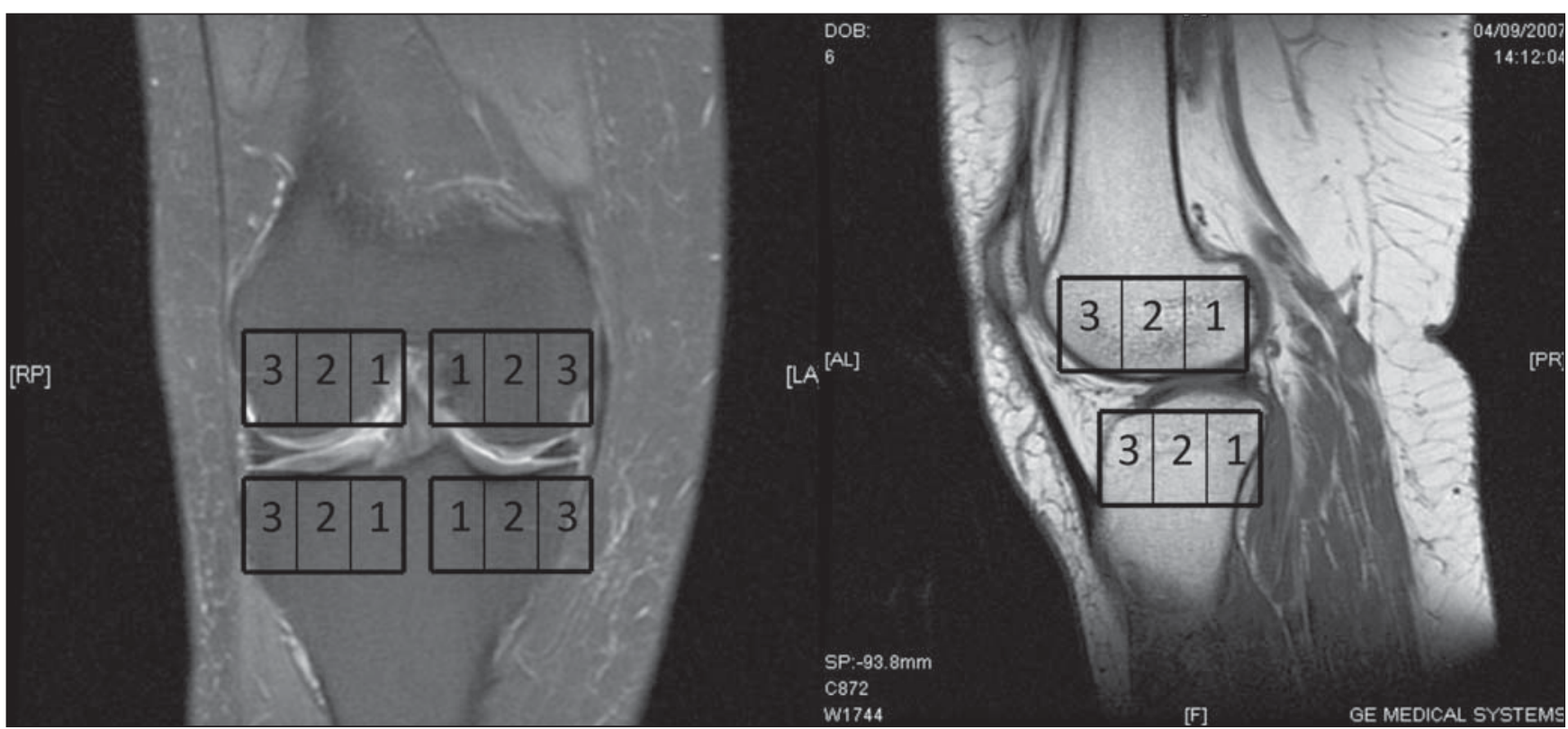

Figura 1. Imagens nos planos coronal (DP FSE) e sagital (T1 FSE) demonstrando a divisão por zonas das epífises ósseas. 


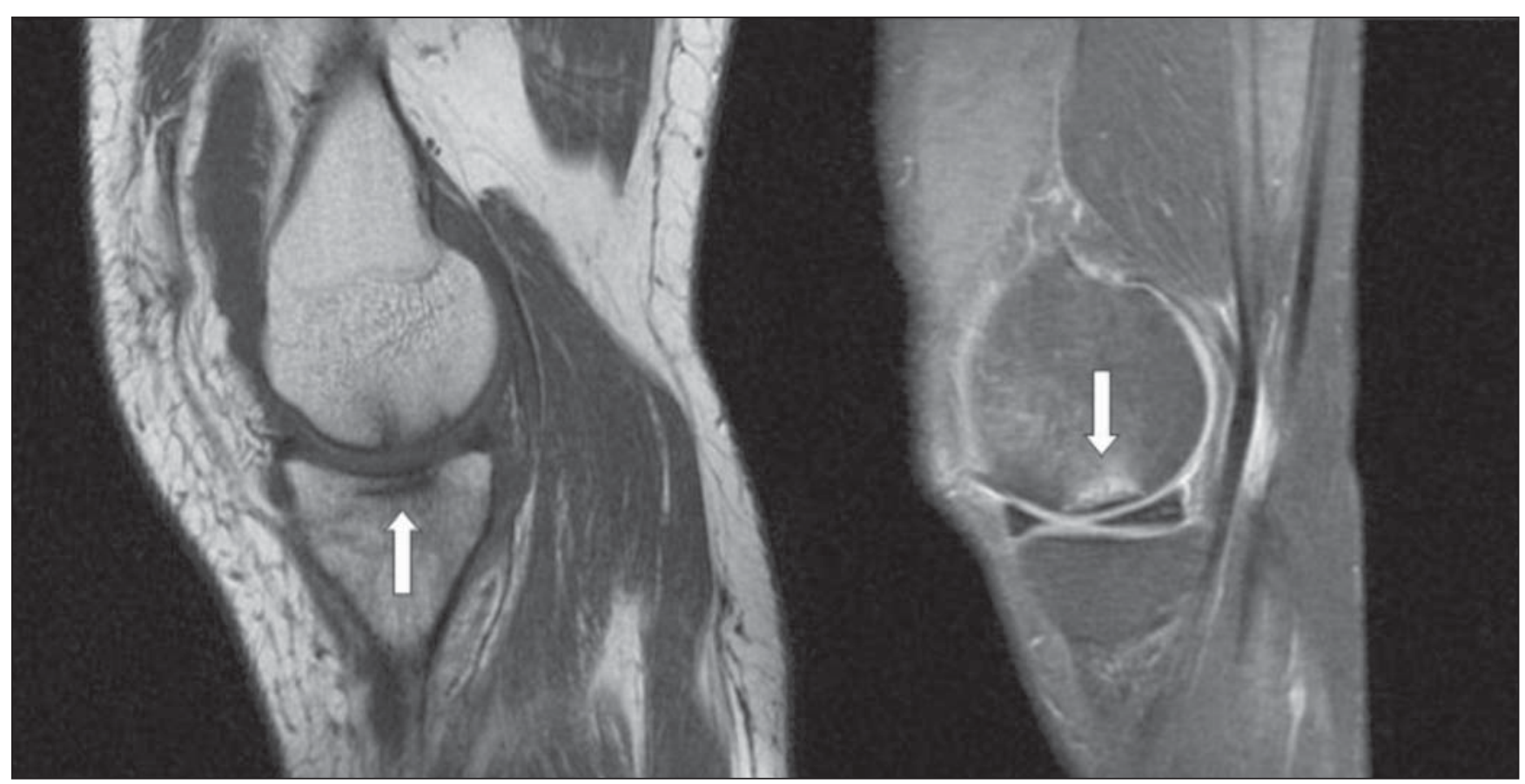

Figura 2. Imagens no plano sagital (T1 FSE / DP FSE) mostrando a anormalidade linear subcondral (setas).

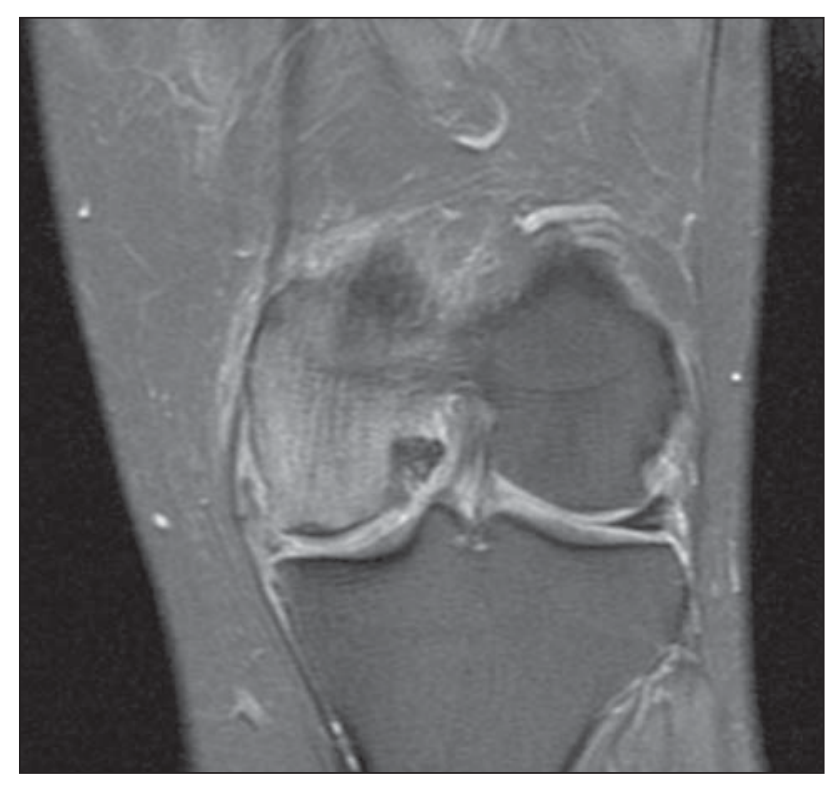

Figura 3. Imagem no plano coronal (DP FSE) mostrando edema grau III na medular óssea do côndilo femoral medial (área com sinal hiperintenso).

(eixo lateral), 16,8 mm (eixo ântero-posterior) e $11,8 \mathrm{~mm}$ (eixo transversal) e volume médio estimado em $466 \mathrm{~mm}^{3}$.

Em $63,1 \%$ dos pacientes foram encontradas anormalidades condrais, ao passo que em 14 casos $(73,6 \%)$ se constatou a presença de lesão meniscal ipsilateral associada, com critérios de instabilidade (lesão radial, lesão do ligamento raiz) em 6 $(31,5 \%)$ destes.

\section{DISCUSSÃO}

A osteonecrose espontânea do joelho é uma afecção que acomete majoritariamente indivíduos do sexo feminino, notadamente a partir da sétima década de vida ${ }^{(5)}$, sem associação com desordens sistêmicas, abuso de álcool, terapia prévia com corticosteroides, cirurgia meniscal ou trauma local. Ahlbäck et al. ${ }^{(6)}$, na primeira descrição que temos conhecimento, estudou 40 pacientes (6 do sexo masculino e 34 do sexo feminino), com média de idade de 70 anos. Em trabalho realizado por Eschard et al. ${ }^{(2)}$, a distribuição pelo sexo demonstrou clara predominância do gênero feminino (23 casos contra 11 do sexo masculino). $\mathrm{Na}$ nossa amostra, de um total de 19 pacientes, $12(63,1 \%)$ eram mulheres e $7(36,9 \%)$ eram homens, com média de idade de 61 anos.

Lotke e Ecker ${ }^{(7)}$ definiram como critérios clínicos para o diagnóstico de osteonecrose o início súbito de gonalgia acentuada em pacientes acima de 60 anos, sem relato associado de trauma. Segundo Eschard et al. ${ }^{(2)}$, o início agudo de dor, ao contrário da literatura geral, apareceu em apenas $25 \%$ da incidência esperada. No presente estudo, a grande maioria dos pacientes avaliados relatou, na época da realização dos exames de ressonância magnética, início súbito de dor localizada.

Na descrição inicial de Ahlbäck et al. ${ }^{(6)}$, corroborada pelos estudos de Lotke e Ecker ${ }^{(7)}$, o sítio de acometimento mais comumente encontrado para as anormalidades osteocondrais foi o côndilo femoral medial. Na série de Björkengren et al. ${ }^{(\mathbf{8})}$, esta foi a localização dos achados em todos os 16 casos. Da mesma maneira, no 
nosso trabalho houve discreto predomínio de comprometimento do côndilo femoral medial, seguido pelo platô tibial interno.

Estudos prévios já enfatizaram a associação de osteonecrose espontânea com lesão meniscal, e não sendo o diagnóstico da doença em questão realizado ao submeter o referido paciente a procedimento cirúrgico de meniscectomia, a aceleração da deterioração do quadro e, consequentemente, do acometimento articular, são evidentes. De acordo com Muscolo et al. ${ }^{(9)}$, nos cinco casos avaliados com lesão meniscal houve posterior aparecimento de anormalidades osteocondrais ipsilaterais. Assim, corroborando a literatura, houve considerável predominância de casos com lesão meniscal homolateral associada às lesões osteocondrais, mais frequente nas com critérios de instabilidade.

Devido à baixa sensibilidade e a um valor de verossimilhança negativo alto no diagnóstico das lesões condrais pela ressonância magnética ${ }^{(\mathbf{1 0})}$, a ausência de achados por este exame de imagem não exclui a existência daquelas. No presente estudo, em mais da metade dos casos $(63,1 \%)$ foram constatadas anormalidades condrais associadas, valor que pode ser inferior em comparação com a artroscopia, considerada método diagnóstico padrão ouro.

Alguns trabalhos ${ }^{(4,11)}$ já relacionaram a presença de fratura por insuficiência, em ossos previamente "enfraquecidos", como um desencadeador do processo da osteonecrose, a partir de correlação, entre outros fatores, com achados semelhantes descritos para a osteonecrose da cabeça femoral. Yamamoto e Bullough ${ }^{(\mathbf{1})}$ relataram achados histológicos, postulando que um dos even- tos primários que induziriam ao desenvolvimento da osteonecrose seria uma fratura subcondral por insuficiência do microtrabeculado ósseo, em pacientes com ossos "mecanicamente" enfraquecidos por doenças não tumorais, especificamente a osteoporose, a despeito de qualquer confirmação com medidas de massa óssea dos pacientes estudados. No trabalho realizado por Ramnath e Kattapuram ${ }^{(4)}$ foi proposto que a presença de um sinal "linear" subcondral na ressonância magnética associado às anormalidades osteocondrais já descritas afirmaria tal relação, enquanto a ausência deste sugeriria maior associação com achados de osteoartrose, colocando em xeque a validade do termo utilizado atualmente ("espontânea"). Notou-se, ainda, nos pacientes da nossa amostra, o início súbito da dor, preferencialmente nas zonas de sustentação de peso, além de um maior grau de edema na medular óssea associado. Já em pacientes sem tal característica, o início da dor seria insidioso e as anormalidades osteocondrais seriam mais evidentes, ao contrário do edema subcondral, mais discreto nestes casos. Na grande maioria dos pacientes analisados no nosso estudo foi demonstrado o sinal "linear" subcondral, bem como uma maior associação da presença deste com edema, em comparação aos casos sem tal alteração.

\section{CONCLUSÃO}

A ressonância magnética demonstrou ser um método não invasivo com boa sensibilidade no diagnóstico da osteonecrose do joelho, bem como das lesões associadas, sendo mais frequente nas mulheres $(63 \%$ dos casos), demonstrando, ainda, a associação da anormalidade linear subcondral com o quadro clínico de gonalgia súbita e grau acentuado de edema na medular óssea adjacente.

\section{REFERÊNCIAS}

1. Yamamoto T, Bullough PG. Spontaneous osteonecrosis of the knee: the result of subchondral in sufficiency fracture. J Bone Joint Surg Am. 2000; 82:858-66.

2. Eschard JP, Brochot P, Etienne JC. Osteonecrosis of the femoral condyles: a retrospective study of 34 cases. Eur J Orthop Surg Traumatol. 1997;7: 267-70.

3. Muglia VF, Simão MN, Elias Júnior J, et al. Erros comuns de interpretação de ressonância magnética de joelho: como reconhecê-los e evitá-los. Radiol Bras. 2001;34:161-6.

4. Ramnath RR, Kattapuram SV. MR appearance of SONK-like subchondral abnormalities in the adult knee: SONK redefined. Skeletal Radiol. 2004;33:575-81.

5. Lotke PA, Ecker ML, Alavi A. Painful knees in older patients: radionuclide diagnosis of possible osteonecrosis with spontaneous resolution. J Bone Joint Surg Am. 1997;59:617-21.

6. Ahlbäck S, Bauer GCH, Bohne WH. Spontaneous osteonecrosis of the knee. Arthritis Rheum. 1968;11:705-33

7. Lotke PA, Ecker ML. Current concepts review. Osteonecrosis of the knee. J Bone Joint Surg Am. 1988;70:470-3.

8. Björkengren AG, AlRowaih A, Lindstrand A, et al. Spontaneous osteonecrosis of the knee: value of MR imaging in determining prognosis. AJR Am J Roentgenol. 1990;154:331-6.

9. Muscolo DL, Costa-Paz M, Ayerza M, et al. Medial meniscal tears and spontaneous osteonecrosis of the knee. Arthroscopy. 2006;22:457-60.

10. Karam FC, Silva JLB, Fridman MW, et al. A ressonância magnética para o diagnóstico das lesões condrais, meniscais e dos ligamentos cruzados do joelho. Radiol Bras. 2007;40:179-82.

11. Kattapuram TM, Kattapuram SV. Spontaneous osteonecrosis of the knee. Eur J Radiol. 2008;67: 42-8. 